\title{
Breaking the Boundaries of Aerial networks with Charging Stations
}

\author{
Prodromos-Vasileios Mekikis* and Angelos Antonopoulos ${ }^{\dagger}$ \\ *Iquadrat Informática S.L, Barcelona, Spain \\ ${ }^{\dagger}$ Centre Tecnològic Telecomunicacions Catalunya (CTTC/CERCA), Castelldefels, Spain \\ e-mail: vmekikis@iquadrat.com, aantonopoulos@cttc.es
}

\begin{abstract}
Considering that cities are typically divided in residential and business districts, massive population migrations between different areas introduce considerable discrepancies in the traffic distribution. However, although novel technologies are constantly introduced to fulfill the $5 \mathrm{G}$ requirements, the current cellular networks raise some limitations due to their static deployment. Aerial networks with unmanned aerial vehicles (UAVs) that carry access points can flexibly overcome this issue by controlling this traffic effectively in an ad hoc manner, but their limited power storage is their major drawback. In this paper, we propose the use of solar-powered charging stations to satisfy the energy needs of UAVs and formulate a theoretic matching problem to ensure the best performance in terms of energy, communication, and safety. Then, we evaluate the network performance by comparing the coverage and utilization between the two scenarios: i) the static infrastructure, and ii) the aerial network, and we present useful insights regarding the benefits of aerial networks.
\end{abstract}

Index Terms-Aerial networks, Unmanned Aerial Vehicles, Matching theory, Stochastic geometry, Charging stations, Tidal traffic, Performance evaluation

\section{INTRODUCTION}

Fulfilling the requirements of the $5 \mathrm{G}$ wireless systems has been a significant ambition for the Information and Communications Technology (ICT) industry and academia during the last few years. Novel concepts and technologies are constantly introduced to achieve higher data rates and spectral efficiency with significantly reduced latency [1]. In this way, it is gradually becoming possible to handle the unprecedented amount of traffic that is expected to appear in the next few years [2]. However, all these technologies are still based on a static infrastructure that introduces some major limitations in the network performance, energy efficiency and deployment cost [3]. More specifically, while some areas suffer from traffic congestion during the day, other parts of the network infrastructure are underutilized [4].

Regarding the aforementioned issue, some traffic patterns have been observed in cities that divide the area into several functional districts, like business and residential districts, where the population massively migrates between them causing traffic discrepancies [4]. For instance, during the day the business areas are more crowded, while the opposite happens during the night with higher traffic in the residential areas. This phenomenon, also known as tidal traffic effect, poses a crucial issue to the network operation and management of large cities, as the network traffic is no longer distributed uniformly. In order to account for this rapidly growing issue, the network designer can ensure higher coverage through small cell (SC) densification [5]. Nevertheless, a dense SC network, is even more poorly utilized in places where the "tide" is low, leading to excessive energy and deployment costs for these places without any additional benefit during the low traffic hours.

To account for these issues, recent studies proposed the deployment of access points (APs) mounted on unmanned aerial vehicles (UAVs) [3], [6]. This kind of APs is expected to be a crucial part of the future communication systems as they are flexible, fast, autonomous, and able to provide a drastic solution to the aforementioned issues. Moreover, in contrast to the underutilized SCs in the static infrastructure scenario, UAVs can follow the actual traffic needs at a given moment or even deploy proactively based on information deducted by techniques such as crowdsensing [7]. Therefore, by employing UAVs instead of SCs we can have a highly utilized network that is able to follow the traffic and, thus, offer higher coverage and reduced handover delays. However, in order to take advantage of the aerial networks characteristics, we should be able to cope with their energy needs, since the limited amounted of power that can be stored on a UAV and their high energy needs are a significant drawback that should be addressed.

Recent studies on UAV networks have identified the power issue in UAV networks [8], [9]. More specifically, the authors in [8] investigate energy-efficient designs for UAV communication through UAV trajectory optimization by jointly considering the communication throughput and the propulsion energy consumption of the UAVs. Although the paper provides interesting and important results that increase the network energy-efficiency, their proposal does not offer a complete solution that will provide a sustainable UAV network. An interesting proposal to cope with this issue could be the use of specialized solar-powered charging stations (CSs). These stations could be distributed in strategic places around the city in the same manner that SCs are deployed, and offer an unobstructed operation in which UAVs never pause their operation. Instead, whenever a UAV is out of power it could be recharged at its nearest CS, while maintaining its service. Moreover, as a CS can provide charging docks to more than one UAV, their intensity could be far lower than the SC intensity, saving in infrastructure cost, as each CS could be able to charge several UAVs at the same time and be responsible for even more. Furthermore, to increase the network 
sustainability, energy harvesting can be employed at the CSs to provide a cost-effective solution [10], [11]. Nevertheless, as the tidal traffic patterns will force the UAVs to change location often, there is a need to assign each UAV to a CS optimally. The existence of multiple operators stress the need for a distributed and self-organizing techniques. Towards this direction, matching theory [12] has recently emerged as an effective solution for such kind of problems, where some resources have to be assigned appropriately to increase the benefits for all participants. Matching theory is a powerful tool to study the formation of mutually beneficial relations among different types of rational and selfish agents, i.e., in our case the aerial network and CS operator. Hence, a matchingtheoretic assignment problem has to be formulated to find a solution to this practical problem of this complex network.

To that end, in this paper, we propose the use of UAV networks to confront the tidal traffic issue that is deepened due to the rapid urbanization, making it an important performance issue for network operators. In order to avoid the inherent power issues of the UAVs, we propose the use of a uniformly distributed set of CSs that are able to charge UAVs in their area of operation. For the allocation of the UAVs to the respective $\mathrm{CS}$, we employ matching theory to guarantee the highest possible profit for all operators. Therefore, our contribution can be summarized as follows:

- We provide a novel network architecture where UAVs aided by solar-powered CSs can serve the entire area of operation in a sustainable way.

- We introduce a matching theoretic algorithm to solve the UAV allocation problem, based on the current network traffic status and safety parameters.

- We provide a performance evaluation analysis on the coverage and utilization and compare UAV and SC scenarios.

The rest of the paper is organized as follows. Section II describes the system model. Section III presents the matching theory analysis, while Section IV includes the performance comparison. Finally, Section V concludes the paper.

\section{System MOdeL}

The system model of our work is introduced in this section and it is presented from four different angles. First, we discuss the network topology and the various entities that compose the architecture of the two scenarios, i.e., SC and UAV. Then, we provide the communication model and establish the channel characteristics that define the data transmission and reception between the UEs and the APs. Next, as the UAV recharging is an essential part of our proposal, we provide the energy model for the UAVs and the CSs, and, finally, we discuss about the business model of this architecture.

\section{A. Network Model}

We consider a wireless network, deployed on the Euclidean plane, that is divided into two districts, i.e., residential and business. As seen in Fig. 1, we model this characteristic by assuming a residential area in the north of the plane and a business area in the south of it. Also, we assume two scenarios (i.e., SC and UAV) involving four types of entities:
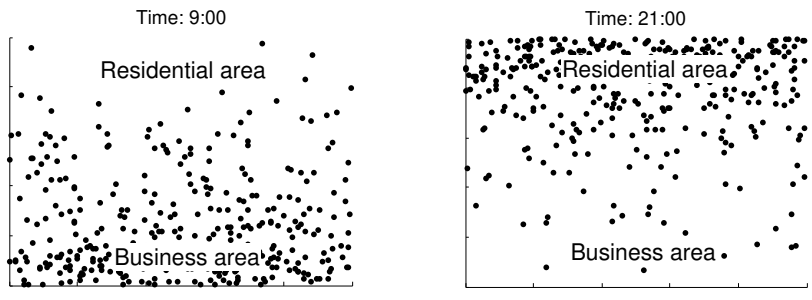

Fig. 1: UE distribution of the same area during the day (left) and the night (right).
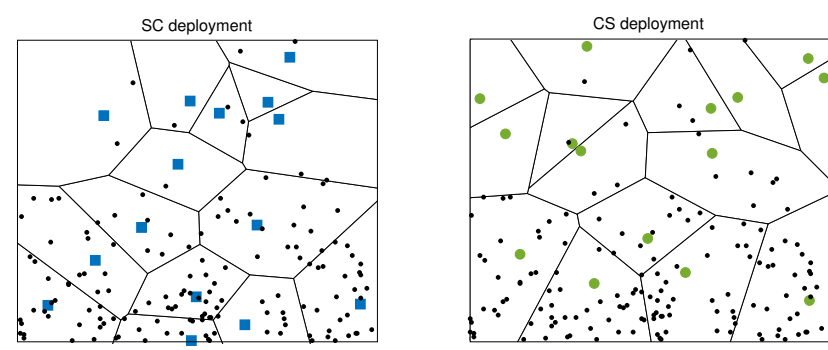

Fig. 2: Network topology for the two scenarios for the same UE deployment: i) SC deployment (blue squares), and ii) CS deployment (green circles).

- User Equipment devices (UEs): In both scenarios, there are $N$ UEs deployed in the network under investigation. Due to the tidal traffic characteristics, the UEs are uniformly distributed in the horizontal dimension and exponentially distributed in the vertical (across the different districts from north to south). Depending on the hour of the realization, the UE density in each district changes accordingly, as seen in Fig. 1. Moreover, each UE can have different types of traffic, i.e., Voice call, Video streaming or HD Video streaming.

- Small cells (SCs): For the SC scenario, there are $M$ uniformly distributed SCs on the same plane with the UEs. The positions of the SCs define a Voronoi tessellation, and we assume that each UE is associated to the SC of the respective Voronoi cell in which it falls in. Also, each SC is capable of providing coverage to a maximum number of $N_{\max }$ UEs, based on realistic SC characteristics. The number of UEs in the area of an SC is denoted with $N_{S C}$, while the number of the UEs that are served by an SC is denoted by $S_{S C}$.

- Unmanned Aerial Vehicles (UAVs): For the UAV scenario, we assume that, instead of SCs, there are $U$ UAVs on the same three-dimensional space with the UEs. Their purpose is identical with that of the SCs, but they have the additional advantage of being able to relocate and hover at a height $H$ over certain areas, based on the current network status. Each UAV carries an access point (UAVAP) that can serve a maximum number of $N_{\max }$ UEs, similarly to the SCs. Moreover, the UAVs are powered through a limited capacity battery that needs to be safely recharged during their operation. To that end, we employ charging stations that recharge the UAVs whenever their battery is nearly depleted. The UAVs are required to 
operate in the vicinity of these stations to avoid power outages.

- Charging stations (CSs): For the UAV scenario, we assume that there are $C$ CSs responsible for the UAVcharging, that are deployed by following a uniform distribution. Similarly to the SC deployment, the positions of the CSs define a Voronoi tessellation. Each CS has the capacity to charge $q_{C S}$ UAVs simultaneously. To that end, we assume that at most $q_{C S}$ UAVs will be associated to a certain CS and will be deployed inside its Voronoi cell. These UAVs will then serve the UEs that fall in the same Voronoi cell defined by the CS. The number of UEs in a CS cell is denoted with $N_{C S}$, while the number of the UEs that are served by the UAVs located in the cell defined by the CS is denoted by $S_{C S}$.

In Fig. 2, we demonstrate the network topology during the day (09:00 AM). As it can be seen, in both scenarios, there is low traffic in the top and high traffic in the bottom of the plane. Therefore, the UAVs should be associated accordingly to the CSs in order to handle the heterogeneous traffic distribution. In this way, higher number of UAVs could cover the high traffic area providing more efficient coverage. On the other hand, in the SC deployment where the topology is fixed, the SCs on the top might be underutilized, while the bottom SCs might not be able to handle the excessive traffic.

\section{B. Communication Model}

We examine the ability of an AP (SC or UAV-AP) to provide coverage at the UEs, based on the signal-to-interference-plus noise at the UEs: $\operatorname{SINR}=\frac{r^{-\alpha} h P_{t} G}{I+W}$, where $P_{t}$ is the AP transmission power, $W$ is the thermal noise of the receiver, $r$ is the distance between the AP and a UE, $\alpha$ is the path loss exponent, $G$ is the directional gain of the AP antenna and $h$ is the fast fading power coefficient, which is independent and identically distributed (i.i.d.). Depending on the requested type of traffic, an AP allocates to the UE a corresponding combination of subcarriers in the time-frequency domain in the form of resource blocks (RBs). The value of the perceived SINR at the receiver, determines the Channel Quality Indicator (CQI) index and, thus, the modulation scheme and the coding scheme (MCS) that will be used by the AP. As it is typical, we assume that the antenna of the SCs is omnidirectional and, thus, it has no directional gain, i.e., $G=1$. On the other hand, the UAV-APs can benefit from the fact that they hover at a height $H$ and employ directional antennas that point directly downwards with antenna half beamwidth $\theta$. Hence, the antenna gain within the main lobe can be approximated by $G_{3 d B}=\frac{29000}{\theta^{2}}$, while outside of the main lobe, it can be considered zero [14].

\section{UAV and CS Energy Model}

In the UAV scenario, the UAVs are equipped with a battery of limited capacity $E C_{U A V}$ and they are recharged by the randomly distributed CSs. We assume that the initial battery energy of each UAV follows a uniform distribution in $\left\{B_{U A V_{m}} E C_{U A V}, B_{U A V_{M}} E C_{U A V}\right\} \subseteq\left\{0, E C_{U A V}\right\}$, where $B_{U A V_{m}}$ and $B_{U A V_{M}}$ are the minimum and maximum battery level percentage (i.e., $0 \%$ and 100\%), respectively. For safety reasons, each UAV must be positioned in the Voronoi cell defined by its assigned CS during the networking operation.

Moreover, each CS is equipped with a battery of total capacity $E C_{C S}$. For the charging operation, CSs employ highpower inductive chargers, similar to those used for electric vehicles [13]. Furthermore, the CSs are connected to a solar panel of size $A \mathrm{~m}^{2}$ for energy harvesting. If the panel of a CS has harvested enough solar energy, its assigned UAVs will be charged through the battery. To avoid any risk and to increase the UAV scenario reliability, the CSs are also connected to the electricity grid in case there is not enough energy in the battery. Consequently, if the battery cannot satisfy the energy needs of the assigned UAVs, they will be charged through the grid with a higher cost, as it will be explained in the following section. It should be mentioned that the battery-level-status of all CSs is shared in the network to inform the UAVs about the status of each CS.

\section{Business Model}

In our model, we assume that there are two operators: i) the network operator, and ii) the CS operator. More specifically,

- Mobile network operator (MNO): The network operator is responsible for the cellular network connectivity and for providing high quality service to its customers (i.e, the UEs) using either SC or UAV deployments. For the SC scenario, the goal of the MNO is to provide the highest possible coverage to the UEs and have a profit from these services. In many cases of this scenario, it is not always possible to provide the best achievable service, as tidal traffic might create unpredictable traffic patterns that a static infrastructure cannot follow.

- CS operator (CSO): The CS operator exists only in the UAV scenario and is the owner of the CS deployment. The recharging cost depends on the amount of harvested solar energy in the battery. Therefore, there are two different prices for the UAV recharging: i) The MNO will pay $p_{s}$ to the CSO if the energy originates from the battery, and ii) The MNO will pay $p_{g}$ to the CS operator if the energy originates from the electricity grid. Hence, the main objective of the CSO is to maximize its profit by charging the UAVs with the lowest battery level.

\section{A College Admission Game for Charging STATION SELECTION}

\section{A. Game Formulation}

In this section, we will describe the proposed matching game in detail. As multiple UAVs will be assigned to each $\mathrm{CS}$, we will use the framework of college admission matching games, also known as many-to-one matching games. In this framework, a college is considering a set of $n$ applicants, among which admits a quota of merely $q$. Having evaluated their qualifications, the admissions office must decide which ones to admit based on the preferences of both sides. Hence, this game assigns applicants (UAVs) to colleges (CSs) in the most satisfactory way for both parties. This framework is 
suitable for our proposed problem as it can provide a stable and desirable association for the aerial network, while increasing the profit and ensuring a more secure flight operation. To that end, we formulate a college admissions game referred to as the CS admissions game, defined by three components: i) the set of UAVs acting as students, ii) the set of CSs acting as colleges, and, iii) preference relations for the CSs and the UAVs allowing them to form preferences over one another.

Definition 1. In a UAV network, the UAV-CS matching game is defined by two finite sets of players, the set $\mathcal{C}$ of $C S s$ and the set $\mathcal{U}$ of UAVs, and a function $\phi:\{\mathcal{C} \cup \mathcal{U}\} \rightarrow\{\mathcal{C} \cup \mathcal{U}\}$, such that the following conditions are met:

(i) $|\phi(c)| \leq q_{C S}, \forall c \in \mathcal{C}$

(ii) $|\phi(u)|=1, \forall u \in \mathcal{U}$

(iii) $\forall c, u \in \mathcal{C} \cup \mathcal{U}, c \in \mathcal{U}$ if and only if $u \in \mathcal{C}$.

The first condition of Definition 1 expresses that CSs do not get associated with more UAVs than they can accommodate (i.e., $q_{C S}$ ) on their charging $\operatorname{spot}(\mathrm{s})$. Assuming a matching $\phi(c)=\left(u_{1}, c\right), \ldots,\left(u_{X}, c\right)$ of CS $c$ with $X$ UAVs, such that $X<q_{C S}$, a CS $c$ can accommodate $\left(q_{C S}-X\right)$ more UAVs. In the set $\mathcal{C} \cup \mathcal{U}$, each $C S$ appears as many times as the number of the obtained UAVs. The second condition represents the fact that each UAV is associated exclusively with one CS. Moreover, the third condition expresses that the matching is bilateral, namely a UAV is associated with a CS if and only if the CS obtains this UAV.

The outcome of the proposed matching game is a matching of CSs and UAVs that allows CSs to be associated with a number of UAVs equal or lower than their quota $q_{C S}$, while each UAV can be matched with one CS at most in each matching period $t$. In this UAV allocation problem, a stable matching must be achieved. For this purpose, the concept of pairwise stability is employed [15].

Definition 2. In a UAV network, the UAV-CS matching game is characterized as pairwise stable if it is not blocked by any player (UAV or CS) or any UAV-CS pair.

A pairwise stable UAV allocation is the state where there exist no subset of the players (CSs or UAVs) that can select sets of partners preferred by all players of the subset, by establishing coalitions only among the players of the subset. For pairwise stability, two conditions have to be met: i) individual rationality and ii) responsiveness.

Out of all possible UAV allocations that may arise during the matching process, only some of them are acceptable by the involved players. The CSs accept the allocations that allow them to serve the maximum possible number of UAVs. The UAVs are interested in establishing partnerships with the CSs until all of their resources are occupied. The matching that produces acceptable outcomes holds the property of individual rationality [16]:

Definition 3. A matching $\phi$ is individually rational if each $U A V$ and CS prefers its current match over being unmatched.

A matching is not individually rational, when either a UAV is not accepted by a CS or a CS is not acceptable by a UAV.
The property of responsiveness is related to the way the preferences of CSs over UAVs are formed [16]. Regarding the responsive preferences of $\mathrm{CS} c$ we can define that:

Definition 4. For any set $\mathcal{U}^{\prime} \subset \mathcal{U}$ and any $U A V u$ and $u^{\prime}$ that are not in $\mathcal{U}^{\prime}, C S$ c prefers $U^{\prime} \cup u$ to $U^{\prime} \cup u^{\prime}$ if and only if $u$ is preferred to $u^{\prime}$, and prefers $U^{\prime} \cup u$ to $U^{\prime}$ if and only if $u$ is acceptable.

The responsiveness of the CSs' preferences denotes that the CSs rank each UAV individually in order to obtain sets of UAVs. Otherwise, they should express preferences over each element of the set of the subsets of acceptable UAVs. A CS $c$ always prefers to add an acceptable UAV, until the quota $q_{C S}$ is reached and prefers replacing a UAV with a better one, e.g., a UAV that is closer or has lower battery life.

\section{B. UAV and CS Preferences}

The goal of UAVs is to serve as many UEs as possible, in order to maximize their profit. In this way, they will also increase the network coverage and the QoS of the UEs. To calculate the profit of a single UAV, we need to take into account its relative position to each CS, the number of UEs in the cell defined by each CS and the battery level of the UAV and the CSs. Therefore, the objective $O_{U A V}$ of the matching game is to maximize the profit for every UAV $u$ in a given CS $c$, defined as

$$
O_{U A V}(u, c)=\frac{e_{U A V} N_{U E}(c) t}{q_{C S}}-g_{c}(u, c),
$$

where $N_{U E}$ is the number of UEs located inside the cell formed by $\mathrm{CS} c, e_{U A V}$ is the profit that a UAV makes by serving one UE, $t$ is the time duration before the next matching takes place, and $g_{c}$ is the distance-dependent charging cost, which is given by

$$
g_{c}(u, c)=\left(1-\frac{B_{U A V}(u)}{100}+\frac{B_{t r}(u, c)}{E C_{U A V}}\right) p_{g},
$$

where $B_{t r}(u, c)=d(u, c) m_{t}$ is the percentage of battery consumed during the UAV transition, with $d(u, c)$ being the distance between a UAV $u$ and a CS $c$, and $m_{t}$ being the consumed energy per meter while the UAV transits from one location to another. Also, $B_{U A V}$ and $B_{C S}$ denote the current battery level of a UAV or a CS, respectively. In this way, (2) takes into account the current battery levels of both the CS and the UAV, to calculate accurately the potential cost to charge UAV $u$ at this specific CS, while taking into account the power that it will consume during its transit to this CS.

Regarding CSs, their goal is to maximize their profit, while ensuring safety and fast response. Thus, they prefer the UAV located at the nearest point to them given that it is safe for this UAV to reach the location of the CS. Therefore, the objective $O_{C S}$ of their matching game is defined as

$$
O_{C S}(c, u)=\frac{\mathbb{1}\left(B_{U A V}(u)-B_{t r}(u, c)>B_{\text {limit }}\right)}{d(u, c)},
$$

where $\mathbb{1}$ is the indicator function, while $B_{\text {limit }}$ is the lower battery limit allowed to ensure safe transit and charging. Using 
(1) and (3), the preference lists among each UAV and CS are calculated and sorted. These lists will be used as an input to the matching game procedure.

Based on the previous analysis, the UAV allocation problem can be formulated as a profit maximization problem, which is directly related with the number of UEs that are being served. Therefore, the gain of this matching can be maximized with the following problem:

$$
\begin{aligned}
\max & \left(\sum_{c=1}^{C} \sum_{N=1}^{S_{C S}(c)}\left(t e_{U A V}\right)+\sum_{u=1}^{U} g_{c}\right) \\
\text { s.t., } & B_{U A V}(u)-B_{t r}(u, c)>B_{\text {limit }}, \forall u \in \mathcal{U}, \\
& N_{U A V} \leq q_{C S}, \\
& S_{C S}(c)<q_{C S} N_{\text {max }}
\end{aligned}
$$

\section{Proposed Algorithm}

To calculate the UAV-CS matching for the problem in (4), we propose Algorithm (1), inspired by the deferred acceptance scheme applied for the college admissions game, which was first suggested by Gale and Shapley [12].

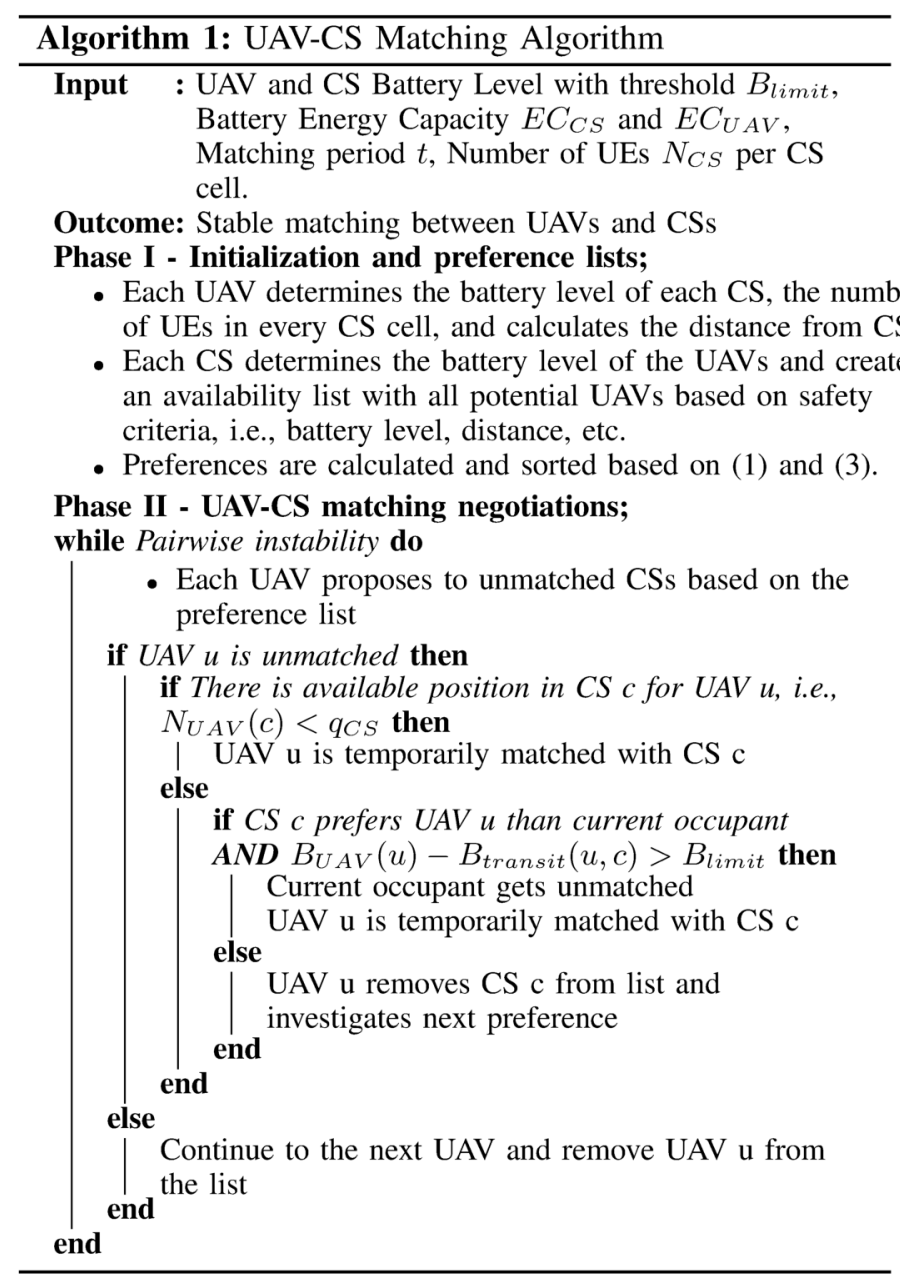

\section{Analytical and Simulation Results}

In this section, we validate the proposed theoretical framework via extensive simulations and provide useful insights
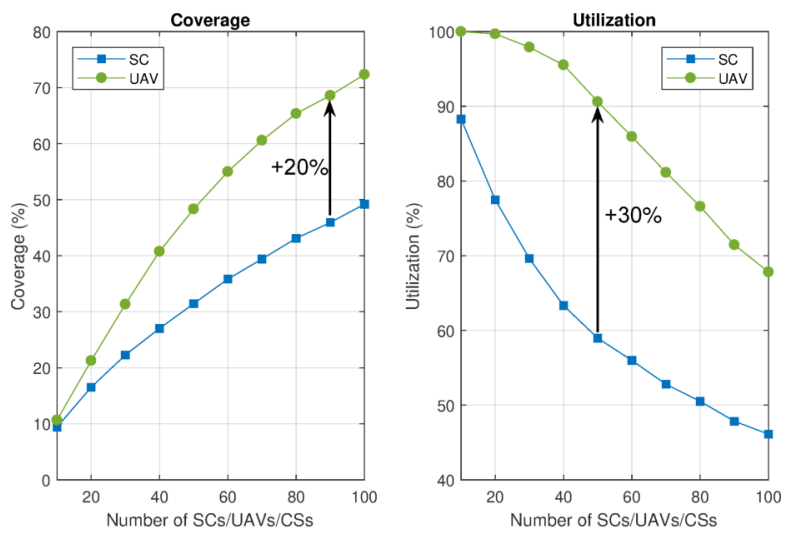

Fig. 3: Coverage and Utilization comparison for the two scenarios

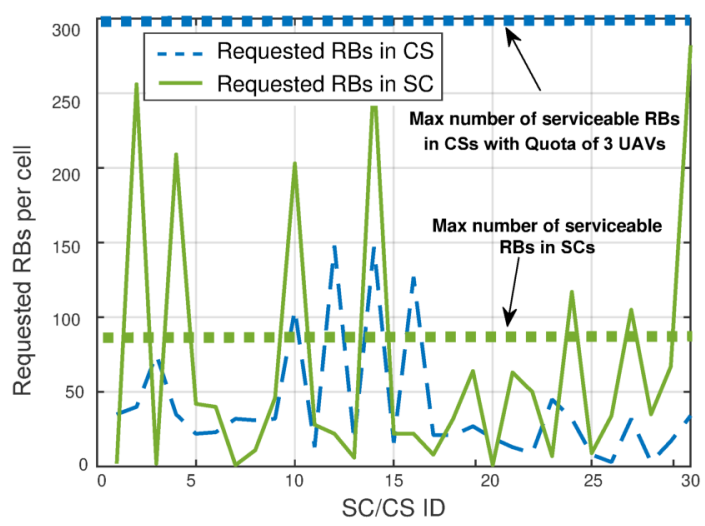

Fig. 4: Requested RB comparison for the two scenarios

on the performance of SC and UAV networks by comparing the metrics of interest for the two scenarios. The simulation environment is developed in Matlab R2016a.

\section{A. Simulation Setup}

We consider the topology described in Section II and evaluate the performance of the aerial network compared to the static SC network in a simulation area of $10^{6} \mathrm{~m}^{2}$. The number of SCs, UAVs and CSs varies from 10 to 100 and they attempt to serve 3000 UEs. In the UAV scenario, the CSs have a quota of 2 to 3 UAVs and they charge them using a battery with capacity $1500 \mathrm{Wh}$. The UAVs have a battery with smaller capacity at $150 \mathrm{Wh}$ and carry an AP with transmission power that varies from $26 \mathrm{dBm}$ to $36 \mathrm{dBm}$, similar to the SCs. Each AP is able to provide $100 \mathrm{RBs}$. Since the UAV Antenna has a certain half beamwidth, i.e., $80^{\circ}$, the directional gain within the main lobe can be approximated by $G_{3 d B}=\frac{29000}{\theta^{2}}=4.5$ [14]. Regarding the path-loss exponent, we employ $\alpha=4$ as it is a fitting value for urban environments, while the thermal noise for is fixed at $-174 \mathrm{~dB} / \mathrm{Hz}$.

\section{B. Performance Evaluation and Comparison}

In Fig. 3, we depict the performance of the two scenarios in terms of coverage and utilization as the number of devices (i.e., SCs, UAVs and CSs) increases. As expected, increasing 


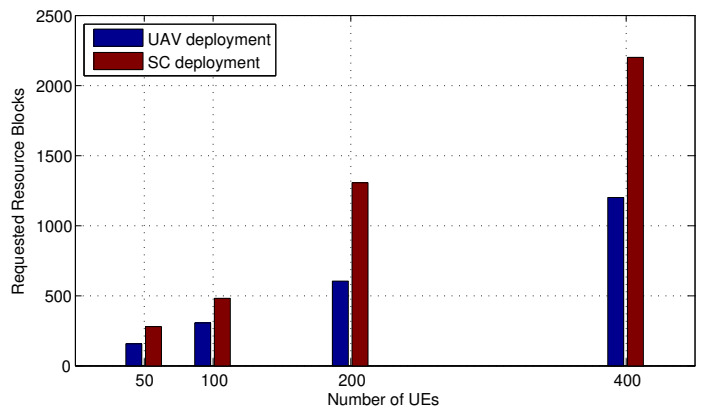

Fig. 5: Requested RBs per UE population

the number of APs in the area, provides better coverage for the UEs in both scenarios. However, in the UAV case, the total network coverage is higher, as the UAVs can follow the traffic and densify on-demand the APs to provide higher number of RBs in congested areas. On the other hand, in a static SC deployment, it is obviously not possible to predict and satisfy congestions at certain locations, resulting in a much lower coverage probability ( $20 \%$ less coverage for 100 SCs). It should be noted that some SCs located in low-traffic districts will not be able to provide their resources, while in other districts the traffic will be extensive with the SCs being unable to serve it. Without doubt, this affects heavily the SC utilization as it can be also seen in Fig. 3. From this figure, we notice that the UAVs can have much higher utilization (up to $30 \%$ higher), because they are strategically located using our matching algorithm in areas where they are most needed. The decrease of utilization as the number of UAVs is rising happens due to the fact that there are CS cells in which the UEs will reasonably require less RBs than the maximum available from one AP (i.e., $100 \mathrm{RBs}$ ) and, thus, the UAV will not utilize all of its available RBs. Still, the UAV scenario presents a remarkable performance in terms of utilization.

To demonstrate a more in-depth analysis of the network performance in each scenario, we present in Fig. 4 the number of requested RBs in each SC (green line) and CS (blue line), assuming there are $30 \mathrm{SCs}$ and $30 \mathrm{CSs}$ in the area. The number of requested RBs in the SC scenario is much higher in many cases. This occurs due to the fact that the UAVs can provide line-of-sight communication and their antennas have directional gain that results in higher SINRs at the UE receivers. Therefore, it is reasonable for the UEs to request lower numbers of RBs as their coding rate is higher. Moreover, in this figure, we present the max number of serviceable RBs in a SC cell, which is 100 as there is only one SC per SC cell, while for the UAV scenario it is 300 , as we assume a quota of maximum three UAVs in the cell defined by the CS. However, due to the higher UAV connectivity, the maximum number of requested RBs in a CS cell does not exceed 150, meaning that there is no need in this certain case to have a quota of three UAVs, as two UAVs can cover the UEs in all cells. Hence, even with a quota of one UAV per CS, the coverage in most cases would be much higher compared to the SC scenario as they can offer higher SINRs at the UEs, while the SCs struggle to satisfy the much higher demand. This result can be furthered stressed in Fig. 5, in which we observe that as the number of UEs in a SC/CS cell is increasing, the SC deployment requires much higher capacity to satisfy the demand. It is interesting to notice that for the CS deployment and 400 UEs the requested $\mathrm{RBs}$ are less than the requested RBs in an SC cell when it is occupied by 200 UEs.

\section{CONClusion}

So far, aerial networks have been praised by academia and industry for their flexibility to provide coverage in any given area. However, their power issue still hinders actual research and development in great scale. In this paper, we proposed the use of solar-powered charging stations that can overcome the power issue and provide a safe UAV operation. As UAVs change location often to cover traffic discrepancies in the various districts, we employed matching theory to ensure safety and energy efficiency. Then, we evaluated the performance of the UAV scenario compared to a static network with SCs and demonstrated that UAVs can provide a much more flexible deployment with higher coverage and utilization.

\section{ACKNOWLEDGEMENTS}

This work has been funded by AGAUR (2017-SGR-891) and the research projects SEMIoTICS (780315), and SPOT5G (TEC2017-87456-P).

\section{REFERENCES}

[1] A. Gupta and R. K. Jha, "A Survey of 5G Network: Architecture and Emerging Technologies," IEEE Access, vol. 3, pp. 1206-1232, 2015

[2] Ericsson, "Ericsson Mobility Report 2018," June 2018.

[3] S. Hayat, E. Yanmaz and R. Muzaffar, "Survey on Unmanned Aerial Vehicle Networks for Civil Applications: A Communications Viewpoint," IEEE Commun. Surveys \& Tutorials, vol. 18, no. 4, pp. 2624-2661, 2016.

[4] Z. Niu, "TANGO: traffic-aware network planning and green operation," IEEE Wireless Communications, vol. 18, no. 5, pp. 25-29, October 2011.

[5] N. Bhushan et al., "Network densification: the dominant theme for wireless evolution into 5G," IEEE Commun. Magazine, vol. 52, no. 2, pp. 82-89, February 2014.

[6] P.-V. Mekikis et al., "Communication recovery with emergency aerial networks," IEEE Trans. Consumer Electronics, vol. 63, no. 3, pp. 291299, Aug. 2017.

[7] K. Ota et al., "QUOIN: Incentive Mechanisms for Crowd Sensing Networks," IEEE Network, vol. 32, no. 2, pp. 114-119, Mar.-Apr. 2018.

[8] Y. Zeng and R. Zhang, "Energy-Efficient UAV Communication With Trajectory Optimization," IEEE Trans. Wireless Commun., vol. 16, no. 6, pp. 3747-3760, Jun. 2017.

[9] M. Hua et al., "Power-Efficient Communication in UAV-Aided Wireless Sensor Networks," IEEE Commun. Letters, vol. 22, no. 6, pp. 1264-1267, June 2018.

[10] P. Mekikis et al., "Connectivity Analysis in Clustered Wireless Sensor Networks Powered by Solar Energy," IEEE Trans. Wireless Commun., vol. 17, no. 4, pp. 2389-2401, Apr. 2018.

[11] H. Li, K. Ota, M. Dong, et al., "Energy Cooperation in Battery-Free Wireless Communications with Radio Frequency Energy Harvesting," ACM Trans. Embedded Computing Systems, vol. 17, no. 2, Apr. 2018.

[12] D. Gale and L. S. Shapley, "College Admissions and the Stability of Marriage," Amer. Math. Monthly., vol 69, no. 1, pp. 9-14, Jan. 1962.

[13] Ch. P. Ricci, "Electric vehicle charging station system and method of use." U.S. Patent 9,944,192 B2, issued April 17, 2018.

[14] C. Balanis, "Antenna Theory: Analysis and Design," Wiley Interscience, NY USA, 2005.

[15] A. E. Roth, "Deferred Acceptance Algorithms: History Theory Practice and Open Questions," International Journal of Game Theory, vol. 36, no. 3-4, pp. 537-569, 2008.

[16] A. Roth, M. Sotomayor, "Two-sided Matching: A study in GameTheoretic Modeling and Analysis," no. 18, Cambridge Univ. Press, 1992. 